\title{
How to Measure Superheavy Spectra
}

\section{A proposed technique could allow researchers to measure spectra of} elements above atomic number 102, despite the tiny quantities in which they are produced.

\section{By Dan Garisto}

$\mathrm{M}$ easuring the spectrum of an atom allows researchers to learn about its structure, but many superheavy elements are produced in such small quantities that these experiments are impossible. Now a proposed spectroscopy technique may solve this problem. The method, called laser resonance chromatography (LRC), involves exciting ions with a laser and then measuring their drift times in a gas-filled tube to determine their quantum states. The technique would be more efficient in its use of the limited number of ions than conventional approaches. Researchers plan to use it to make the first spectral observations of lawrencium (element 103) and potentially of other superheavy elements.

Each line in an atom's spectrum corresponds to a photon that is emitted or absorbed when an electron jumps from one energy level to the next. These spectral lines are unique to each element and can be used to infer electronic, nuclear, and chemical properties of atoms. Superheavy elements are not a

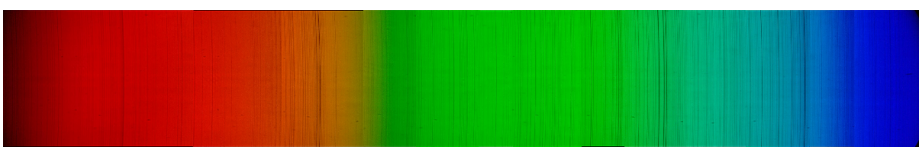

All of the colors. This solar spectrum contains dark absorption lines associated with each of the Sun's elements, such as the two strong sodium lines in the orange range. A new technique could allow researchers to measure spectra for the heaviest known elements. Credit: Flickr/yellowcloud precisely-defined category, but the term often refers to those above nobelium (element 102). High-precision, superheavy spectra could provide a wealth of information, such as details of nuclear properties, which could help theorists improve models of the long-predicted (but never observed) group of superheavy isotopes known as the "island of stability."

Researchers have relied on resonance ionization spectroscopy (RIS) to measure elements up to nobelium. In RIS, superheavy ions from a beam must first be neutralized-given additional electrons-so that they can later be excited and ionized in a controlled manner. Unfortunately, neutralization results in the loss of some atoms from the experiment, which makes RIS unsuitable for the tiny quantities in which the heaviest elements are produced.

To measure these atoms, Mustapha Laatiaoui of the GSI Helmholtz Centre for Heavy Ion Research in Germany and his colleagues propose LRC, a technique that doesn't require neutralization and thus avoids the loss of atoms that accompanies this step. "I would like to have the ions as-is," says Laatiaoui. "I get them as ions; I would like to keep them as ions. I don't want to have an intermediate step of neutralization."

In the proposed setup, superheavy ions from an accelerator are hit with a laser that sends some of the ions into excited electronic states. The ions then travel through a cryogenic drift tube filled with helium gas until they reach a silicon detector. An ion's travel time through the gas depends on the number of collisions with helium atoms, which is strongly affected by its electronic configuration. So ground-state ions can be 
distinguished from excited ions based on their arrival times at the detector. By varying the laser frequency and monitoring the ratio of the number of ground-state to excited-state ions, researchers could build up a spectrum.

The team uses the term "chromatography" to describe the experiment because in chemistry the word refers to methods for separating components of a mixture based on the speed with which they move through paper or a column of beads, for example. In LRC, ions are distinguished by their drift times through helium gas.

Several researchers agree that the method seems feasible, since it borrows from existing protocols. "This idea has a lot of potential," says Francesca Giacoppo, a nuclear physicist at GSI
Helmholtz who was not involved with the research. "This really gives us a chance to go much higher [in atomic number], to investigate many other atomic species." Simon Sels, a nuclear physicist at CERN in Geneva, says that the experimental setup is carefully designed. "It should do the trick," he says.

Laatiaoui is in the process of building the setup at the University of Mainz in Germany, hoping to use the technique to measure a spectrum of lawrencium, but he says that other chemists or physicists could possibly measure it before him, now that the method is published.

This research is published in Physical Review Letters and Physical Review A.

Dan Garisto is a freelance science writer based in New York. 